\title{
Relationships Between Perceived Features and Similarity of Images: A Test of Tversky's Contrast Model
}

\author{
Abebe Rorissa \\ Department of Information Studies, University at Albany, State University of New York, Draper Hall, Room 113, \\ 135 Western Avenue, Albany, NY 12222. E-mail: arorissa@albany.edu
}

The rapid growth of the numbers of images and their users as a result of the reduction in cost and increase in efficiency of the creation, storage, manipulation, and transmission of images poses challenges to those who organize and provide access to images. One of these challenges is similarity matching, a key component of current content-based image retrieval systems. Similarity matching often is implemented through similarity measures based on geometric models of similarity whose metric axioms are not satisfied by human similarity judgment data. This study is significant in that it is among the first known to test Tversky's contrast model, which equates the degree of similarity of two stimuli to a linear combination of their common and distinctive features, in the context of image representation and retrieval. Data were collected from 150 participants who performed an image description and a similarity judgment task. Structural equation modeling, correlation, and regression analyses confirmed the relationships between perceived features and similarity of objects hypothesized by Tversky. The results hold implications for future research that will attempt to further test the contrast model and assist designers of image organization and retrieval systems by pointing toward alternative document representations and similarity measures that more closely match human similarity judgments.

\section{Introduction}

People from all walks of life as well as organizations produce and/or use images for their daily activities, including business, medical treatment, education, and entertainment, to name a few. Getty Images, Inc. (http://www.gettyimages.com) has over 70 million still images in its collection, and serves over 2.3 billion thumbnails to its 2.3 million unique visitors a month. The rapid growth of the numbers of images and their users as a result of the reduction in cost and increase in efficiency of the creation, storage, manipulation, and transmission

Received February 1, 2006; revised October 16, 2006; accepted October 16, 2006

(C) 2007 Wiley Periodicals, Inc. • Published online 00 XXXXXX 2007 in Wiley InterScience (www.interscience.wiley.com). DOI: 10.1002/asi.20606 of images poses challenges to those who organize and provide access to them. One of these challenges is similarity matching (to determine interdocument similarity or the degree of similarity of representations of users' queries and documents), a key component of content-based image retrieval systems, and visualization of both stored and retrieved sets of images. These are often implemented through similarity measures based on geometric/spatial models of similarity (Zachary, 2000)

Similarity plays an important role in human perception (Melara, 1992; Tversky, 1977; Tversky \& Gati, 1978) and information organization and retrieval (Qin, 2000; Santini \& Jain, 1999; Zhang \& Korfhage, 1999a, 1999b; Zhang \& Rasmussen, 2001). Even though geometric/spatial models of similarity are widely utilized as similarity measures, Tversky (1977) identified some weaknesses in their metric assumptions/axioms (e.g., the fact that human similarity judgment data violate the metric axioms) and, consequently, formulated an alternative set-theoretical model known as the contrast model. A brief review of the major psychological models of similarity, including the contrast model, and similarity measurement in image retrieval is discussed next.

\section{Psychological Models of Similarity}

Similarity is one of the most important and well-researched constructs (Goldstone, 1999; Tversky, 1977). It "refers to the outcome of a comparison among entities, usually a comparison based on many of the entities' properties. Objects are similar to the degree that they have features in common and do not have distinctive features" (Sloman \& Rips, 1998, p. 4). According to Tversky (1977), similarity "serves an organizing principle by which individuals classify objects, form concepts, and make generalizations" (p. 327). People organize, group, and categorize things based on their degree of similarity and separate them based on their degree of difference or dissimilarity. What makes two things similar has been a focus of several investigations in psychology, cognitive and behavioral sciences, and related fields for over 100 years (Melara, 1992). Through these investigations, a number of 
theories and models have been formulated and tested to explain perceived similarity (Attneave, 1950; Shepard, 1962a, 1962b; Thurstone, 1927; Tversky, 1977).

From around 1850, psychophysicists such as Fechner started to study the nature of human similarity judgments through investigations of relationships between physical and psychological changes. By creating the "just noticeable difference" or jnd, a scale to measure psychological change, Fechner laid the foundations for scaling or measurement of similarity relations (Melara, 1992), thereby laying the foundations for the earliest model of similarity, based on the idea that jnd is a fixed entity. This assumption was challenged by Thurstone (1927), who argued that a human observer gives "different comparative judgments on successive occasions about the same pair of stimuli" (p. 274). This led to the formulation of his law of comparative judgment, another classic model of similarity, which defines similarity relations as probabilistic. Even though the two classic models of similarity pioneered by Fechner and Thurstone did not at first have a big impact in explaining similarity relations, they definitely influenced the scaling of similarity of stimuli as well as the development of multidimensional scaling (Melara, 1992).

While Fechner's jnd and Thurstone's Law of Comparative Judgment "examined the psychological properties of a single dimension of experience," multidimensional scaling "allowed an investigator to determine how many psychological dimensions subjects used when judging similarity" (Melara, 1992, p. 316). The geometric/spatial models of similarity, more specifically multidimensional scaling, are based on the assumption that the multidimensional space, on which the stimuli are represented as points in the space, is metric.

Given three points $x, y$, and $z$ in a multidimensional space and a metric distance function $\delta$, a metric space is one that satisfies the three metric axioms/conditions (Tversky, 1977), namely:

- Positivity/Minimality: $\delta(x, y) \geq \delta(x, x)=0$ (The distance between $x$ and $y$ is zero if they are identical and positive if they are distinct.);

- Symmetry: $\delta(x, y)=\delta(y, x)$ (The distance from $x$ to $y$ is equal to the distance from $y$ to $x$.); and

- Triangle Inequality: $\delta(x, z) \leq \delta(x, y)+\delta(y, z)$ (The distance between $x$ and $z$ is less than or equal to the sum of the distance between $x$ and $y$ and the distance between $y$ and $z$ ).

Even though the idea of representing stimuli as points in a multidimensional space is attractive, it requires a mechanism by which distances between the points are determined. While it is easier to know what the dimensions of simple perceptive stimuli are and to determine, through experiments, how these dimensions combine to estimate overall similarity of stimuli (Attneave, 1950), this is not the case with more complex stimuli. Attneave (1950) limited his search for metrics or rules for determining the distances between the points in the multidimensional space, and hence the overall similarity of the stimuli represented by those points, to the Minkowski family of metrics. The two metrics chosen by Attneave were the Euclidean and the city-block or Manhattan metrics, two metrics which later became among the most popular in similarity measurement for text and image retrieval (Zachary, 2000; Zhang \& Rasmussen, 2001). Given two points, $X$ and $\mathrm{Y}$, in a $p$-dimensional space $(p \geq 1)$ with $\left(\mathrm{X}_{1}, \mathrm{X}_{2}, \mathrm{X}_{3}, \ldots, \mathrm{X}_{\mathrm{p}}\right)$ and $\left(\mathrm{Y}_{1}, \mathrm{Y}_{2}, \mathrm{Y}_{3}, \ldots, \mathrm{Y}_{\mathrm{p}}\right)$ as their respective coordinates, the distance between the two points, in terms of the Minkowski metric, is given by: $d(X, Y)=\sqrt[r]{\sum_{i=1}^{p}\left|X_{i}-Y_{i}\right|^{r}}$. When $r=1$, $d(X, Y)=\sum_{i=1}^{p}\left|X_{i}-Y_{i}\right|$ and it is called the city-block or the Manhattan distance. When $r=2, d(X, Y)=$ $\sqrt{\sum_{i=1}^{p}\left(X_{i}-Y_{i}\right)^{2}}$, and it is the familiar Euclidean distance.

Attneave (1950) is one of the early researchers to notice some of the weaknesses of the geometric/spatial models of similarity. Others have gone even further and have not only formulated alternative models of similarity (Tversky, 1977) but also tested the alternative models under several conditions using a variety of stimuli (Gati \& Tversky, 1984; Tversky \& Gati, 1978, 1982). Even with the introduction of nonmetric multidimensional scaling by Shepard (1962a, 1962b), which made possible the analysis of original similarity judgment data using a single technique through monotonic transformation, geometric/spatial models of similarity did not escape from criticism. Most of the geometric/spatial models assume that humans, in their judgments of similarity and/or dissimilarity of stimuli, pay equal attention to the various dimensions. Shepard (1964) noticed that humans attach unequal weights to different dimensions and suggested that any analysis of similarity judgment data take into account individual differences among humans. This gave rise to a scaling procedure called individual differences scaling (Melara, 1992).

Despite several critics noting the weaknesses of geometric/ spatial models of similarity, these models still remain the dominant and most used (Tversky, 1977; Tversky \& Gati, 1978). Multidimensional scaling is useful because people's visual-spatial experience base is much more strongly horizontal than vertical (i.e., our eyes much more often scan horizontally than vertically). Apart from the geometric/spatial and Tversky's (1977) contrast models, the other two noteworthy models of similarity are the transformational and alignment-based models. The transformational and alignmentbased models are better suited to explaining the nature of similarity of well-structured stimuli. Transformational models are based on the notion that two stimuli are more similar if few numbers of operations are required to make the two stimuli identical, by transforming one of the two (Goldstone, 1999). According to Goldstone (1999), in alignment-based models, "comparing things involves not simply matching features, but determining which elements correspond to or align with one another" (p. 758). In addition, in alignmentbased models, features that align with one another also may need to have similar functions.

The three models of similarity, namely the geometric/spatial (e.g., MDS), transformational, and alignment-based models, may not be well suited to explaining similarity relations of every possible set of stimuli due to weaknesses peculiar to each one of them. The strength of a similarity model should 
be measured, in part, on the basis of how well it explains the nature of human similarity judgments.

\section{The Contrast Model}

In a seminal article, Tversky (1977), in line with Attneave's (1950) argument, not only showed that metric axioms/ assumptions of geometric/spatial models of similarity are not satisfied by human similarity judgments data but also formulated and tested an alternative set-theoretical model of similarity called the contrast model. Tversky's contrast model, unlike geometric/spatial models of similarity which represent stimuli as points in a multidimensional space, defines stimuli as sets of features and the similarity of any two stimuli as a linear function of a measure of their common and unique/distinctive features. Tversky defined a feature or attribute of a stimulus as its components, whether concrete properties or abstract attributes - a definition adopted by this author.

Perhaps the clearest distinction between the geometric/ spatial models of similarity and the contrast model emanates from the fact that while dimensions have mutually exclusive levels (e.g., color has levels of red, green, blue, etc.), features are dichotomies (i.e., a stimulus either has or does not have a particular feature) (Rosch \& Lloyd, 1978). Furthermore, the contrast model posits that two stimuli are more similar if they have more common features and fewer unique/distinctive features. They are less similar if they have more unique/distinctive features and fewer common features. Given two stimuli $a$ and $b$ and their respective feature sets $A$ and $B$, the perceived similarity of $a$ and $b$, denoted by $s(a, b)$, is expressed as a linear function of the measures of their common and unique/distinctive features (Tversky, 1977; Tversky \& Gati, 1978), and is given by: $\mathrm{S}(a, b)=$ $\theta f(A \cap B)-\alpha f(A-B)-\beta f(B-A)$, where:

- $A \cap B$ represents the common features of $a$ and $b$,

- $A-B$ represents features of $a$ that $b$ does not have (i.e., unique/distinctive features of $a$ ),

- $B-A$ represents features of $b$ that $a$ does not have (i.e., unique/distinctive features of $b$ ),

- $\theta, \alpha$, and $\beta$ reflect weights given to the common and unique/distinctive features and are nonnegative $(\theta, \alpha, \beta \geq 0)$,

- $S$ is an interval scale such that $S(a, b)>S(c, d)$ if and only if $s(a, b)>s(c, d)$; that is, $a$ and $b$ are more similar than are $c$ and $d$,

- $f$ is an additive function [i.e., $f(A \cup B)=f(A)+f(B)$ ], whenever $A$ and $B$ are disjoint $(A \cap B=\varnothing)$.

Another form of the contrast model, called the ratio model, is given by: $S(a, b)=\frac{f(A \cap B)}{f(A \cap B)+\alpha f(A-B)+\beta f(B-A)}$, where $\alpha, \beta \geq 0$. The ratio model defines a normalized value of similarity such that $0 \leq S \leq 1$. It is a generalized form of the contrast model (Tversky, 1977). In the earlier equations, the function $f$, apart from being a measure of the common and unique/distinctive features of the stimuli, also is an indicator of the salience or prominence of the stimuli, and factors such as the information content, intensity, frequency, familiarity, and good form of the stimuli contribute to the salience of stimuli (Tversky \& Gati, 1978).

Results of several tests of the contrast model agree that humans focus their attention more on common features when judging similarity than when judging differences of stimuli (Gati \& Tversky, 1984; Johnson, 1981; Tversky, 1977; Tversky \& Gati, 1978), and "it is reasonable to assume that enlarging the measure of the common features increases similarity and decreases difference, whereas enlarging the measure of the distinctive features decreases similarity and increases difference" (Tversky \& Gati, 1978, p. 81).

The main reason for Tversky (1977) to formulate and test the contrast model, as indicated earlier, is the violation of the metric axioms, which are the basis of geometric/spatial models of similarity, by similarity rating data. In support of his argument, Tversky cited the fact that an object may not be recognized as itself all the time. In other words, two identical stimuli may not always be judged to be the same. Tversky provided evidence that the symmetry axiom, even though it is one of the basic assumptions of similarity theories, does not always hold. In his study of 21 pairs of countries, he noted that participants judged the similarity of North Korea to Red China to be greater than the similarity of Red China to North Korea. Tversky attributed this asymmetry to the fact that "the variant [North Korea] is more similar to the prototype [Red China] than vice versa" (p. 328), a notion supported by Rosch (1975). To illustrate how the triangle inequality axiom is violated by similarity relations of three stimuli, he noted that even though Jamaica is similar to Cuba (in geographic location) and Cuba is similar to Russia (in ideology/ politics), that does not make Jamaica and Russia similar.

Tversky (1977) and Tversky and Gati (1978), using both perceptual/visual stimuli (e.g., figures, letters of the alphabet, schematic faces) and semantic stimuli (e.g., countries, vehicles), tested the contrast model and showed that the linear combination of measures of common and unique/distinctive features account for close to $76 \%$ (multiple correlation coefficient, $R=0.87$ ) of the variance in similarity, a result that clearly supports the contrast model and, again, attests to the significance of this research for understanding the nature of human similarity judgments of images using the contrast model as a framework. Several researchers have either tested or used the contrast model as a framework for their studies in marketing and advertising (Johnson, 1981, 1986; Johnson \& Horne, 1988), psychology (Ben-Shakhar \& Gati, 1992; Dopkins \& Ngo, 2001; Gati \& Tversky, 1984), and consumer research (Ulhaque \& Bahn, 1992), with results supporting the generality of Tversky's contrast model, even though some recommended further research to corroborate their results (e.g., Ben-Shakhar \& Gati, 1992).

\section{Similarity Measurement in Image Retrieval}

The main "goal of image-indexing systems is to find a set of images that is similar to the target image for which the user is searching" (Neumann \& Gegenfurtner, 2006, p. 31). An equally important goal is bridging the "semantic gap" between 
physical and perceptual features and similarity (Datta, Li, \& Wang, 2005; Lew, Sebe, Djeraba, \& Jain, 2006; Neumann \& Gegenfurtner, 2006). The majority of computerized systems use the vector-space model of document representation (Zachary, 2000), in which similarity matching is achieved through similarity measures. Rubner (1999), in explaining the function and importance of similarity measures in contentbased image retrieval (CBIR) systems, stated that:

In order for an image retrieval system to find images that are visually similar to the given query, it should have both a proper representation of the images' visual features and a measure that can determine how similar or dissimilar the different images are from the query. (p. 7)

Similarity measures are metrics used to determine the relevance of documents in a collection to queries based on proximity between their feature representations or to determine interdocument similarity. Similarity measures play important roles in both text retrieval (Qin, 2000; Zhang \& Korfhage, 1999a, 1999b; Zhang \& Rasmussen, 2001) and CBIR (Gupta, Santini, \& Jain, 1997; Santini \& Jain, 1999; Zachary, 2000; Zachary, Iyengar, \& Barhen, 2001). While the distance-based similarity measures are the most widely used measures in CBIR systems, the cosine (angle)-based measure has had limited applications in this area (Gupta et al., 1997). This is an indication that geometric/spatial models of similarity, including the cosine measure (albeit to a lesser degree in CBIR), have been predominantly applied in both text and image retrieval compared to the other models. Among the geometric/spatial models of similarity, those that use the two Minkowski metrics, namely the Euclidean distance (or $\mathrm{L}_{2}$ norm) and the city-block distance (or $\mathrm{L}_{1}$ norm), to measure similarity/dissimilarity are more popular in content-based image retrieval systems (Rubner, 1999; Stricker \& Orengo, 1995; Zachary, 2000).

Most CBIR systems use the color feature, shape, and texture of images as the basis for their representation and similarity matching (Datta et al., 2005; Lew et al., 2006; Neumann \& Gegenfurtner, 2006). Moreover, while similarity measures used in text retrieval mainly use term frequency and weights in computing interdocument similarity, CBIR systems rely on the color feature and, to a lesser degree, texture, shape, luminosity, and edges. The image database contains feature vectors of images, which are their feature representations, extracted using appropriate feature-extraction algorithms. When a user submits a query image (to a system using a "query by example" method of retrieval), its feature vector is extracted using the same algorithm as the one used to represent images in the database, and images (or their feature vectors) in the database are evaluated for relevance to the user query through a similarity measure; results of this evaluation are produced as retrieved sets of images (Zachary, 2000).

Lately, similarity measures based on the contrast model (e.g., Santini \& Jain, 1999) have been proposed; however, none of these similarity measures have been based on research that tested corresponding models of similarity using humans.
Despite CBIR systems that use various types of similarity measures, the majority of which are based on the geometric/ spatial models of similarity, to determine interdocument similarity or similarity of documents to users' queries and to provide visualizations of stored and/or retrieved image documents as results of users' queries for human browsing, there are not thorough investigations that compare human similarity judgments of images and the various similarity measures (Gupta et al., 1997; Zhu \& Chen, 2000). Unless retrieved sets of documents are examined by the user and judged for their relevance, there is no direct way of determining how similar two documents are either to each other or the document the user had in mind when submitting the query to the system. Therefore, there is a need for a similarity measure that predicts the relevance and similarity judgments of documents by users (Santini \& Jain, 1999), and a similarity measure based on Tversky's (1977) contrast model seems to fit the criterion.

\section{Problem Statement and Purpose}

The amount of research on representation and retrieval of text documents dwarfs that on representation and retrieval of image documents, which is in turn dominated by contentbased (automatic/machine-based) image retrieval literature (Chu, 2001). Lacking from both sets of literature are detailed investigations of the nature of human similarity judgments of images (Jörgensen, 1995; Santini \& Jain, 1999). Despite the major role similarity plays in human perception (Melara, 1992; Tversky, 1977; Tversky \& Gati, 1978) and information organization and retrieval (Qin, 2000; Santini \& Jain, 1999; Zhang \& Korfhage, 1999a, 1999b; Zhang \& Rasmussen, 2001), the various psychological models of similarity, such as Tversky's (1977) contrast model, have not been used enough to investigate problems in information science. In general, current concept-based image indexing mechanisms hardly rely upon any sort of theoretical foundations (Jörgensen, 2003).

Even though the geometric/spatial models of similarity (especially multidimensional scaling) are widely applied in information retrieval in general and image retrieval in particular to determine the extent of interdocument similarity and for visualizations of sets of stored or retrieved text and image documents (e.g., Rubner, 1999), some weaknesses in their metric axioms (e.g., the fact that human similarity judgment data violate these axioms) have been identified by Tversky (1977). Therefore, because similarity matching is a key component of information retrieval and similarity measures use feature/attribute representation of documents, it is desirable to test similarity models such as Tversky's contrast model that better predict/explain human similarity judgments not only to bridge the gap in the relevant literature but also to explore alternative methods of image indexing and retrieval and adopt them for similarity measurement. Neumann and Gegenfurtner (2006), who called for an investigation into the relationship between features and perceived similarity, stated that "Ideally, content-based image retrieval (CBIR) 
systems should be modeled with respect to the users and return images in good agreement with the perceived similarity" (p. 33). Accordingly, the purpose of this study was to examine the relationships between perceived features of images and their similarity using Tversky's contrast model as the theoretical framework, thereby testing the model, through the following four research questions:

- Which methods measure the common and unique/distinctive features of images well?

- To what extent does the contrast model fit human similarity judgments and features/attributes data for a sample of images?

- What is the relationship between perceived similarity of images, as judged by humans, and their features/attributes identified and described/listed by humans?

- What are the relative weights given to common and unique/distinctive features in human similarity judgments of images?

This study is significant in that it is among the first known to use the contrast model as a framework to investigate perceived features and similarity of images in the context of image representation and retrieval. Results of this study will serve as a foundation for future research that will attempt to further test the model and will inform designers of retrieval systems about similarity measures that match perceived similarity and could be used to formulate alternative similarity measures that mimic perceived similarity. The study also is significant in that its results may challenge the widely accepted notion that documents (both text and image) can be represented as points in a continuous multidimensional space and their similarity computed using distance functions that satisfy the three metric axioms of minimality $[\delta(x, y) \geq$ $\delta(x, x)=0]$, symmetry $[\delta(x, y)=\delta(y, x)]$, and the triangle inequality $[\delta(x, z) \leq \delta(x, y)+\delta(y, z)]$, where $x, y$, and $z$ are three points representing three objects in the multidimensional space and $\delta$ is a metric distance function. Results may support the idea that these documents can be represented as sets of discrete features and that their similarity can be determined based on and with varying weights for their common and unique/distinctive features.

\section{Methods}

Materials

A set of color photographs taken by a number of photographers (O'Connor \& Wyatt, 2004) served as the population of images. A simple random sample of 30 color digital images (Appendix A) was selected from this collection. Due to the specific data-collection technique chosen (paired comparison, which involves $n(n-1) / 2$ pairs of images for a sample of size $n$ ) to solicit similarity judgments of images, the size of the sample of images $(n=30)$ may not be large enough to generalize results of the study to other stock photo or image collections; however, it is still large enough for the results to be valid for similar collections.
Each image was $375 \times 250$ pixels in dimension. To ensure heterogeneity and sufficient variations of features of the sample of images selected randomly from the collection, 15 volunteer participants were asked to list features of the images. Feature data (i.e., list of features) for all 30 images were subjected to analysis of spread (variability), and the Index of Qualitative Variation (IQV), a measure of qualitative variability (Weisberg, 1992) was used. The IQV measure, calculated using the formula: $I Q V=\frac{\left(1-p_{1}^{2}-p_{2}^{2}-\cdots-p_{k}^{2}\right)}{(k-1) / k}$, where $p_{i}$ (for $i$ $=1,2,3, \ldots, k)$ is the proportion of distinct features (i.e., features not shared by others) attributed to image $i$ and where $k$ is the number of images in the sample $(k=30)$. The calculated value of the IQV is 0.99965 , a value close to 1 , which signifies greater diversity among the sample of 30 images.

\section{Participants}

Participants were asked to voluntarily serve as similarity judges of images, through paired comparison (where the degree of similarity of images is rated in pairs), and provide descriptions of images in terms of their features/attributes. A convenience sample of 150 beginning master's students from a school of library and information science at a public university was selected. Convenience sampling can be used for testing models provided that the "model is correctly specified" (Schonlau, Fricker, \& Elliott, 2001, p. 34). The model being tested, Tversky's (1977) contrast model, has a strong and well-documented theoretical foundation and well-defined structural relations among the constructs (i.e., perceived features and similarity).

The sample size of 150 participants is well over the recommended minimum number of participants for correlational studies (Gay \& Diehl, 1992) and structural equation modeling (Schumacker \& Lomax, 1996). Participants were randomly assigned to two tasks (described next), with half of them $(n=75)$ providing similarity judgments and the remaining half $(n=75)$ taking part in the image description task. A similar procedure was used by Dopkins and Ngo (2001) and Tversky (1977) and is assumed to produce feature listing and similarity judgment data that are free of interactive influences (Dopkins \& Ngo, 2001). In terms of the setting for data collection, it was not practical to bring participants to a common room and setting; therefore, they were allowed to complete the tasks on their own using their office or home computers and settings. This could be a potential limitation, although it was not evident from results of internal consistency measures, which were high (Cronbach's $\alpha>$.84).

\section{Procedures}

A number of assumptions were made in conducting the study:

- Perceived similarity, like any perceptual continuum, is scalable (i.e., measurable), and participants "can make consistent quantitative appraisals of their subjective experiences" (Stevens, 1956, p. 5); hence, they are able to judge the 
degree of perceived similarity of pairs of images and produce ratio-level data. Psychometric/psychophysical methods of scaling perceived similarity of images have been used successfully in CBIR system evaluation research (Neumann \& Gegenfurtner, 2006).

- It is possible to elicit verbal and written descriptions of images, and data derived thereof can be used for further analysis to address research questions (Tversky, 1977).

- Magnitude estimation has produced useful results in the investigation of perceived similarity of perceptual stimuli such as images (Nunnally \& Bernstein, 1994; Stevens, 1966), and also has been used by information science researchers with encouraging results for scaling users' perception of relevance (Bruce, 1994; Eisenberg, 1986, 1988) and satisfaction with their information seeking on the Internet (Bruce, 1998).

Image description task. Half of the participants $(n=75)$, randomly assigned to complete the image description task, were involved in the description (i.e., listing of features/ attributes) of the 30 images. An e-mail message, with the URL for the image description task and a unique identifier, was sent to each participant during the Summer 2004 and Fall 2004 semesters. A follow-up e-mail message was sent to participants who did not complete the task after 2 weeks from the date the first e-mail message was sent. Each participant was presented with a Web-based form of each image randomly; that is, no 2 participants saw the 30 images in the same order. The first page displayed instructions for participants on how to complete the task, and to ensure internal validity, a time constraint of $90 \mathrm{~s}$ was enforced within which participants were asked to type as many features/attributes as possible to describe the particular image. Two images (not in the sample) were included at the beginning of the task to familiarize participants with the task.

Similarity judgment task. The traditional paired comparison design (where the degree of perceived similarity of images is rated in pairs) of the 30 images was used to collect similarity judgment data. The other half of the 150 participants, once again randomly assigned to this task, completed the task. An e-mail message, similar to the one sent to participants for the image description task, was sent with a followup message sent 2 weeks after the first one. After reading the instructions for this task, participants were presented with a Web-based form for each of two sets of pairs of the 30 images $[30(30-1) / 2=435$ pairs in each set] and were asked to judge the degree of perceived similarity of pairs of images on a ratio scale using magnitude estimation (Stevens, 1956, 1966, 1975).

There are two designs/forms of tasks involving magnitude estimation: with and without a standard stimulus or a modulus (Engen, 1971; Stevens, 1975). In a task involving magnitude estimation with a standard stimulus or a modulus, participants are presented with the standard stimulus or modulus together with the magnitude estimation of the modulus, usually an integer multiple of 10 , predetermined by the researcher. Participants then are asked to estimate the magnitude, relative to the magnitude of the modulus, of a series of stimuli that vary in intensity of the attribute/continuum being scaled/measured. The second form of magnitude estimation uses no standard stimulus or modulus. Participants are presented with the stimuli in random orders, and are asked to assign numbers to each stimulus proportional to their perceived intensity of the attribute/continuum being scaled. A variation of the second form of magnitude estimation instructs participants to mark a point on a horizontal line of a certain length so that the distance between the beginning of the line and the marked point is equal to the magnitude of the intensity of the attribute/continuum being scaled.

The first method of magnitude estimation (with a modulus) was the first to be used in psychometric scaling; however, it was later found that the choice of a standard stimulus by the researcher interferes with the freedom of participants to produce their own magnitude estimations and that the second method (with no modulus) is preferred as it facilitates unconstrained judgments (Stevens, 1975). Therefore, in this study, a variation of the second method of magnitude estimation (with no modulus) was used where participants used a horizontal line (5 in. long and 1/5 in. thick; in data analysis, 1 in. represents 100 units/numbers) to indicate the degree of their perceived similarity of pairs of images.

Perceived similarity of the first set of 435 pairs of the 30 images was judged by the participants of the similarity judgment task first, and the second set of 435 pairs (obtained by reversing the order of pairs in the first set as well as the order of images in each pair) was judged by the same participants after a mandatory 5 -min break to minimize the fatigue effect due to the large number of pairs of images. Pairs of images were presented in the same order for all participants. The order of pairs of images in both sets of 435 pairs as well as the order of presentation of images in a pair were determined using an optimum order and presentation method for paired comparisons suggested by Ross (1934), who argued that the method eliminates errors due to time and space and that other methods that use random orders are open to such errors. Because the sample size of images for this study is an even number $(n=30), n=31$ was used to produce the pairs as suggested by Ross. Pairs involving Image 31 were dropped. As a familiarization and calibration exercise in magnitude estimation, participants were presented with five lines of varying lengths (2-8 in.) and asked to judge their apparent length. Three practice pairs of images (not included in the sample) also were presented at the beginning of the similarity judgment task.

\section{Operationalization of Variables}

Content analysis was used to analyze data from the image description task where the "word" and "word sense" recording units were used to represent individual features of images. A word sense could be a phrase constituting a semantic unit, including idioms such as "taken for granted" as well as proper nouns such as "the Empire State Building" (Weber, 1990). 
A dictionary based on the recording units was created for the purpose of coding the list of features supplied by participants. As is customary in content analysis, two popular measures of intercoder agreement, percent agreement and Cohen's (1960) $\kappa$ (calculated for the researcher and each of two other coders), were used to measure reliability. Even though reliability is not a sufficient indicator of validity, it is one of the necessary conditions. Content (face) validity is sufficient for most content analyses, and it requires results of the content analysis to be consistent with characteristics of the objects under study (Holsti, 1969). Evaluating the descriptions provided by the participants for overelaborations and long stories ensured the content validity and usefulness of the image description task data. Cronbach's (1951) coefficient $\alpha$ was used to measure the reliability of the similarity judgment task data.

Structural equation modeling, also called covariance structure analysis, is a set of statistical techniques that include path analysis (to study direct/indirect effects), factor analysis (to study measurement models, or how well a set of observed variables measure latent variables/constructs), regression analysis (to study prediction and amount of variance explained), and structure (covariance structure) analysis (to study relationships between latent variables) (Schumacker \& Lomax, 1996). It provides a single comprehensive means for data analysis, especially testing complex theoretical models. Structural equation modeling is appropriate for studies that investigate research questions related to the relationships between latent variables/constructs and when researchers are seeking to test theoretical model fit to sample data. In general, a structural equation model has two parts: the measurement model and the structural model (Schumacker \& Lomax, 1996). Because all four research questions deal with either relationships between latent constructs or the testing of a theoretical model, structural equation modeling as well as correlation and regression analyses were chosen as appropriate sets of statistical techniques.

The path diagram in Figure 1 is a graphical representation of Tversky's (1977) contrast model $[S(a, b)=\theta f(A \cap B)-$ $\alpha f(A-B)-\beta f(B-A)]$ tested in this study where the endogenous (dependent) latent variable, $\operatorname{sim}(\eta)$, is the degree of perceived similarity of Images $a$ and $b[S(a, b)]$; commnfet $\left(\xi_{1}\right)$ is one of the exogenous (independent) latent variables and is a measure of the common features of $a$ and $b$ $[f(A \cap B)]$; distfeta $\left(\xi_{2}\right)$ is the second exogenous (independent) latent variable and is a measure of the unique/distinctive features of $a$ vis-à-vis $b[f(A-B)]$; and distfetb $\left(\xi_{3}\right)$ is the third exogenous (independent) latent variable and is a measure of the unique/distinctive features of $b$ vis-à-vis $a$ $[f(B-A)]$. Table 1 is a summary of the variables, coefficients, and parameters in Figure 1 as well as how each observed variable in the model is measured.

Use of more than one measure of the latent variables ensures construct validity and minimizes measurement error. The dependent latent variable (perceived similarity or sim) has two measures, SIMAB and SIMBA, operationalized using the similarity judgment task described earlier. SIMAB is similarity judgments data (obtained using magnitude estimation)

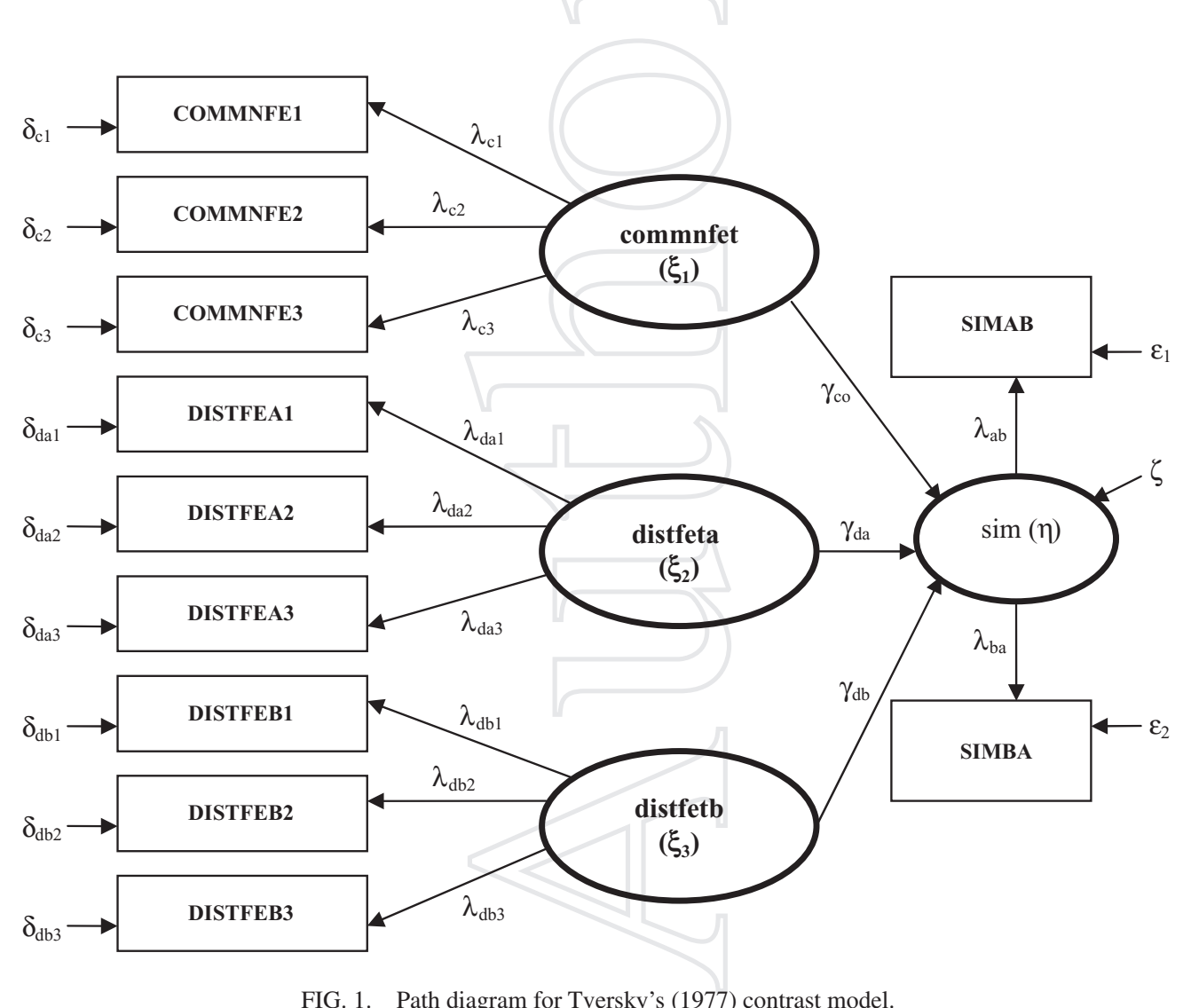

FIG. 1. Path diagram for Tversky's (1977) contrast model. 
TABLE 1. Variables, coefficients, and parameters in Tversky's contrast model (see Figure 1).

\begin{tabular}{|c|c|}
\hline Symbol & Variable, path, or coefficient it stands for \\
\hline$\xi_{1}$ & Common features (commnfet)-exogenous latent variable (1) \\
\hline$\xi_{2}$ & Distinctive features of $a$ (distfeta)-exogenous latent variable (2) \\
\hline$\xi_{3}$ & Distinctive features of $b$ (distfetb)-exogenous latent variable (3) \\
\hline$\eta$ & Perceived similarity ( $\operatorname{sim}$ )-endogenous latent variable \\
\hline$\gamma_{\mathrm{co}}$ & Path coefficient for the path from $\xi_{1}$ to $\eta$ \\
\hline$\gamma_{\mathrm{da}}$ & Path coefficient for the path from $\xi_{2}$ to $\eta$ \\
\hline$\gamma_{\mathrm{db}}$ & Path coefficient for the path from $\xi_{3}$ to $\eta$ \\
\hline SIMAB, SIMBA & Observed y variables (of $\eta$ )-similarity judgments for Image Sets $1 \& 2$, respectively \\
\hline COMMNFE1, COMMNFE2, COMMNFE3 & Observed x variables (of $\xi_{1}$ )-measures of common features using Methods $1,2, \& 3$, respectively \\
\hline DISTFEA1, DISTFEA2, DISTFEA3 & Observed x variables (of $\xi_{2}$ )-measures of distinctive features of $a$ using Methods $1,2, \& 3$, respectively \\
\hline DISTFEB1, DISTFEB2, DISTFEB3 & Observed $\mathrm{x}$ variables ( of $\xi_{3}$ )-measures of distinctive features of $b$ using Methods $1,2, \& 3$, respectively \\
\hline$\lambda_{\mathrm{ab}}, \lambda_{\mathrm{ba}}$ & Path from $\eta$ to SIMAB and SIMBA, respectively \\
\hline$\lambda_{\mathrm{c} 1}, \lambda_{\mathrm{c} 2}, \lambda_{\mathrm{c} 3}$ & Path from $\xi_{1}$ to COMMNFE1, COMMNFE2, \& COMMNFE3, respectively \\
\hline$\lambda_{\mathrm{da} 1}, \lambda_{\mathrm{da} 2}, \lambda_{\mathrm{da} 3}$ & Path from $\xi_{2}$ to DISTFEA1, DISTFEA2, \& DISTFEA3, respectively \\
\hline$\lambda_{\mathrm{db} 1}, \lambda_{\mathrm{db} 2}, \lambda_{\mathrm{db} 3}$ & Path from $\xi_{3}$ to DISTFEB1, DISTFEB2, \& DISTFEB3, respectively \\
\hline$\zeta$ & Error term in the structural equation \\
\hline$\varepsilon_{1}, \varepsilon_{2}$ & Measurement errors in SIMAB \& SIMBA, respectively \\
\hline$\delta_{\mathrm{c} 1}, \delta_{\mathrm{c} 2}, \delta_{\mathrm{c} 3}$ & Measurement errors in COMMNFE1, COMMNFE2, \& COMMNFE3, respectively \\
\hline$\delta_{\mathrm{da} 1}, \delta_{\mathrm{da} 2}, \delta_{\mathrm{da} 3}$ & Measurement errors in DISTFEA1, DISTFEA2, \& DISTFEA3, respectively \\
\hline$\delta_{\mathrm{db} 1}, \delta_{\mathrm{db} 2}, \delta_{\mathrm{db} 3}$ & Measurement errors in DISTFEB1, DISTFEB2, \& DISTFEB3, respectively \\
\hline
\end{tabular}

for the first set of 435 pairs of the 30 images while SIMBA is similarity judgments data for the second set. Like any data obtained using magnitude estimation, values of the two variables (i.e., SIMAB and SIMBA) for the 435 cases (pairs of images) were determined by taking the logarithms of the raw magnitude estimations provided by all participants of the image description task and then aggregated using their geometric means. The three independent latent variables (i.e., common features, unique/distinctive features of $a$, and unique/distinctive features of $b$ ) have three measures each. These measures are three different methods of operationalization (outlined next) of the function $f$ in the contrast model, some of which (Methods $1 \& 2$ ) take into account the number of participants who assign a particular feature to a stimulus.

The three methods of operationalization of the function $f$ (a measure of common and unique/distinctive features) in the contrast model are detailed next.

Method 1. This method, used by Tversky (1977), requires the number of participants who attribute a specific common or unique/distinctive feature to be determined. Let $X_{i}$ be the proportion of participants who assigned Feature $X$ to Image $i$ and $N_{x}$ be the total number of images to which Feature $X$ was assigned. According to Method 1, the measure of the common features of Images $a$ and $b$ is computed using: $f(A \cap B)=\sum X_{a} X_{b} / N_{x}$, for all $X \mathrm{~s}$ in $A \cap B$. Similarly, let $Y_{i}$ and $Z_{j}$ be the proportion of participants who assigned unique/distinctive Features $Y$ and $Z$ to Images $i$ and $j$, respectively. The measures of the unique/distinctive features of Images $a$ and $b$ are $f(A-B)=\sum Y_{a}$ and $f(B-A)=\sum Z_{b}$, respectively, for all $Y \mathrm{~s}$ in $(A-B)$ and all $Z \mathrm{~s}$ in $(B-A)$.
Method 2. According to this method, developed by Johnson (1986), if $C_{i}$ represents the number of common features attributed to Images $a$ and $b$ by Participant $i(i=1,2,3, \ldots, n)$ and $n$ is the total number of participants who assigned at least one common feature to $a$ and $b$, the measure of the common features of Images $a$ and $b$ is the mean number of common features and is given by $f(A \cap B)=\sum C_{i} / n$. To compute the measure of the unique/distinctive features of $a$ and $b$, let $D_{i}$ and $D_{j}$ be the number of unique/distinctive features assigned to Images $a$ and $b$, respectively, by Participants $i(i=1,2,3, \ldots, n)$ and $j(j=1,2,3, \ldots, m)$ and $n$ and $m$ be the total number of participants who assigned at least one unique/distinctive feature to $a$ and $b$, respectively. The measures of the unique/distinctive features of Images $a$ and $b$ are $f(A-B)=\sum D_{i} / n$ and $f(B-A)=\Sigma D_{j} / m$, respectively.

Method 3. This measure, used by Tversky (1977) as well, "assigns equal weight to all features regardless of their frequency of mention" (p. 338). It is determined by simply counting the number of common and unique/distinctive features assigned by participants. Let $C_{i}$ be the number of common features attributed to Images $a$ and $b$ by Participant $i$ $(i=1,2,3, \ldots, n)$ and $n$ be the total number of participants who assigned at least one common feature to $a$ and $b$. The measure of the common features of Images $a$ and $b$ is given by $f(A \cap B)=\Sigma C_{i}$. If $D_{i}$ and $D_{j}$ represent the number of unique/distinctive features assigned to Images $a$ and $b$, respectively, by Participants $i(i=1,2,3, \ldots, n)$ and $j$ $(j=1,2,3, \ldots, m)$ and $n$ and $m$ are the total number of participants who assigned at least one unique/distinctive feature to $a$ and $b$, respectively, the measures of the unique/distinctive 
features of Images $a$ and $b$ are $f(A-B)=\sum D_{i}$ and $f(B-A)=\sum D_{j}$, respectively.

\section{Analysis of Data, Results, and Discussion}

\section{Analysis of Data}

Participants of the image description task $(n=75)$ supplied a total of 15,301 terms in describing the 30 images, an average (mean) of 204 terms per participant and 510 terms per image. The minimum and maximum number of terms assigned to a single image were 352 and 652, respectively. Each participant assigned an average (mean) of 6.8 terms per image. For the final list, 4,133 feature-bearing words and word senses were manually identified and considered for analysis. Appendix B presents all the feature-bearing words and word senses supplied for one of the images.

As described earlier, content analysis was used to build a dictionary of all feature-bearing words and word senses supplied by participants of the image description task. Once the dictionary, with 196 mutually exclusive categories of features, was built by the researcher and three others through consensus, two more coders were asked to assign features to the categories. The two coders examined a random set of features (23\% of the total number of features) and assigned them to 39 randomly selected categories (20\%). The coders were told to leave unassigned the features that did not fit into any of the categories (2.7 and $8.8 \%$ of the sample of 954 features were unassigned by the two coders, respectively). The computed values of the percent agreement and Cohen's (1960) $\kappa$ between the researcher and the two coders were .90 and .92 and .89 and .91 , respectively. These are quite large values, given that reliability coefficients over 0.80 are considered to be "acceptable in most situations" (Neuendorf, 2002 , p. 143). The unit of analysis for all variables in this study, including measures of common and distinctive measures, is pair of images. Values of the measures of common and distinctive features (i.e., values of the variables: COMMNFE1, COMMNFE2, COMMNFE3, DISTFEA1, DISTFEA2, DISTFEA3, DISTFEB1, DISTFEB2, DISTFEB3) for each pair were determined using the three methods discussed earlier.

Similarity judgments (i.e., ratings) by all participants for each pair of images were aggregated using the geometric mean (the appropriate measure of central tendency for such data instead of the arithmetic mean), after log-transformations. According to Stevens (1966, 1975), magnitude estimation data is log-normal, hence the need for log-transformations. Hence, the geometric means of the base-10 logarithms of the magnitude estimations make up values of the two observed $y$ variables, SIMAB and SIMBA, with Cronbach's (1951) $\alpha$ values of .965 and .963 , respectively, which are well above the minimum value of 0.70 often cited in the literature (Nunnally \& Bernstein, 1994).

Prior to any kind of analysis, data need to be checked for missing values, outliers, and univariate normality (Schumacker \& Lomax, 1996). For this study, there were no missing data and no apparent extreme outliers; however, all observed $x$ variables, except three (DISTFEA1, DISTFEA3, DISTFEB1), and both observed $y$ variables, SIMAB and SIMBA, had either skewness or kurtosis or both values significantly different from zero, a property of nonnormal scores (Tabachnick \& Fidell, 1989), prompting the researcher to use transformations of the variables in subsequent analyses. Even though the log-transformation of all observed variables yielded scores with improved skewness and kurtosis values, they still failed to satisfy the univariate normality criterion. Besides, the log-transformed scores have different means and $S D$ s from the original raw scores, and to achieve univariate normality, the raw data were subjected to the "Normal Scores" algorithm in PRELIS (du Toit \& du Toit, 2001; Jöreskog \& Sörbom, 1999), which yielded normal scores for all observed variables.

The other crucial tasks of data screening are checking for pairwise linearity of all variables using scatter plots and checking for convergent validity. An examination of the scatter plots for all pairs of the observed variables showed that they are linearly related. To establish convergent validity, observed variables or measures of a particular latent construct need to correlate (Nunnally \& Bernstein, 1994). All relevant Pearson's product-moment correlation coefficients are significant $(p<.001)$, hence the convergent validity is satisfied.

\section{Results and Discussion}

Extent to which various methods measure the common and distinctive features of images. The first research question is "Which methods measure the common and distinctive features of images well?" Three measures have been reported in the literature (Johnson, 1986; Tversky, 1977) and discussed earlier in this article. The main purpose of this research question is to determine how well the three methods measure the three independent latent variables. This was assessed, using output from LISREL, through measurement model fit indices and loadings of those measures (or estimated parameters called $\lambda^{(x)} \mathrm{s}$ ) on corresponding independent latent variables/ constructs (i.e., common and distinctive features). A covariance matrix consisting of all observed $x$ and $y$ variables (three observed $x$ variables for each of the independent latent variables and two observed $y$ variables, SIMAB and SIMBA, for the dependent latent variable) was produced using LISREL.

A single LISREL program (using the mle method) produced results of tests of model fit of the measurement models for the three independent latent variables measured using the three methods. How well a given observed variable measures a latent variable or construct is dependent on the statistical significance of the relevant loadings or parameter estimates (Schumacker \& Lomax, 1996), provided the measurement model fits the sample data well. The main goal of assessing model fit is to see whether the model produces the original sample covariance matrix (of observed variables) with minimum or no residuals. This is achieved through examination of several model fit indices. To assess model fit, several fit 
indices (i.e., criteria) are recommended instead of a single index since there is no single best index (Schumacker \& Lomax, 1996). Consequently, the most widely used indices such as the chi-square $\left(\chi^{2}\right)$, the Standardized Root Mean Square Residual (SRMR), the Root Mean Square Error of Approximation (RMSEA), the goodness-of-fit index (GFI), and the Adjusted-goodness-of-fit index (AGFI) were used to assess the fit of the measurement models to sample data.

$\chi^{2}$ is a measure of overall model fit and "measures the distance (difference, discrepancy, deviance) between the sample covariance (correlation) matrix and the fitted covariance (correlation) matrix" (Jöreskog, 1993, p. 308). The larger the value of the $\chi^{2}$, the worse the model fit to the data because a larger value of $\chi^{2}$ is an indication of a significant discrepancy between the sample covariance (correlation) matrix and the reproduced (or model implied) covariance (correlation) matrix. The RMR is a measure of the mean difference between the sample (or observed) and the reproduced (model implied) covariance (correlation) matrices. It is the square root of the mean of the squared differences (residuals) between the sample (observed) and the implied covariance (correlation) matrices. The SRMR is preferred to the RMR because it is a standardized summary of average covariance discrepancies, and its values lie between zero and 1. A value closer to 1 is an indication of a good fit (Kelloway, 1998). The RMSEA, which is a "measure of discrepancy per degree of freedom" (Jöreskog, 1993, p. 310), is a model fit measure that takes the population error of approximation into account. The degrees of freedom for a model with $k$ parameters to be estimated and $q$ observed variables is $q(q+1) / 2-\mathrm{k}$.

The GFI and the AGFI were developed as alternatives to the $\chi^{2}$, which is " $\mathrm{N}-1$ times the minimum value of the fit function" and it is affected by $N$, the sample size (Jöreskog, 1993, p. 309). Both the GFI and the AGFI "do not depend on sample size explicitly and measure how much better the model fits compared with no model at all" (Jöreskog, 1993, p. 309), and their values range between zero and 1, with values closer to 1 indicating better fit of the model to the data. The acceptable levels or thresholds of these model fit indices are a $\chi^{2}$ value that is nonsignificant (i.e., the observed and estimated covariance matrices need to be found to not be statistically different), values of RMSEA and SRMR below 0.05 , and values of GFI and AGFI of at least $0.90(\mathrm{Hu} \&$ Bentler, 1999; Jöreskog, 1993).

Table 2 shows values of the model fit indices for the measurement models of common and distinctive features using all observed $x$ variables (Measurement Model 1), and pairs of observed $x$ variables (Measurement Models 2-4). Measurement Model 1 (all observed $x$ variables) was proposed, based on the measures of common and distinctive features reported in the literature on the contrast model, when the contrast model was specified (Figure 1); however, none of the values of the fit indices were below or above the recommended thresholds, $\chi^{2}=548.48, d f=27, p<.01, R M S E A=.211$, $S R M R=.125, G F I=.781, A G F I=.635)$. Since the measurement model with all nine observed $x$ variables is not a good fit to the data, the model had to be modified. Three modified measurement models with pairs of the observed $x$ variables for each independent latent variable were considered. Measurement Model 2 (using observed $x$ variables measured using Methods 2 and 3) was the first to be tested. Once again, the model fit indices for this modified model showed a poor fit, $\chi^{2}=136.24, d f=9, p<.01, R M S E A=.180, S R M R=$ $.0435, G F I=.905, A G F I=.779$ ).

It is evident from Table 2 that measurement models with a combination of observed $x$ variables measured using Methods 1 and 3 (Model 3: $\chi^{2}=7.78, d f=4, p>.05, R M S E A=.047$, $S R M R=.0207, G F I=.994, A G F I=.969)$ and Methods 1 and 2 (Model 4: $\chi^{2}=4.75, d f=4, p>.3, R M S E A=.021$, $S R M R=.0234, G F I=.996, A G F I=.981)$ fit the data well. Among the two, Measurement Model 4 fits the data slightly better. Measurement models that have observed $x$ variables measured using Methods 2 and 3 together consistently did not fit the data well. This appears to be due to multicollinearity, which is a characteristic of two or more measures of the same latent construct (or predictors of the same dependent variable in regression analysis) that are highly correlated, with correlations above .90 , and due to singularity, which is a characteristic of two or more measures of the same latent construct that are perfectly correlated (Tabachnick \& Fidell, 1989). The correlation between DISTFEA 2 and DISTFEA3, measures of distinctive features of $a$ (where $a$ and $b$ form a pair) measured using Methods 2 and 3 is .998 while the correlation between DISTFEB2 and DISTFEB3 is 1.00. The source of this multicollinearity and singularity is the fact that observed $x$ variables measured using Method 2 are simply observed $x$ variables measured using Method 3 divided by the number of participants who attributed the common (for measures of common features) or distinctive (for measures of distinctive features) features to pairs of images or to individual images. Despite the fact that Measurement Models 1 (all observed $x$ variables) and 2 (observed $x$ variables measured using Methods 2 and 3) did not fit the sample data well, all loadings of the observed $x$ variables onto their

TABLE 2. Model fit statistics for measurement models of common and distinctive features.

\begin{tabular}{|c|c|c|c|c|c|c|c|}
\hline Model & $\chi^{2}$ & $d f$ & $p$ & RMSEA & $S R M R$ & $G F I$ & $A G F I$ \\
\hline Measurement Model 1 (all observed $x$ variables) & 548.48 & 27 & .00 & .211 & .125 & .781 & .635 \\
\hline Measurement Model 2 (observed $x$ variables measured using Methods $2 \& 3$ ) & 136.24 & 9 & .00 & .180 & .0435 & .905 & .779 \\
\hline Measurement Model 3 (observed $x$ variables measured using Methods 1 \& 3 ) & 7.78 & 4 & .0998 & .047 & .0207 & .994 & .969 \\
\hline Measurement Model 4 (observed $x$ variables measured using Methods 1 \& 2) & 4.75 & 4 & .3138 & .021 & .0234 & .996 & .981 \\
\hline
\end{tabular}



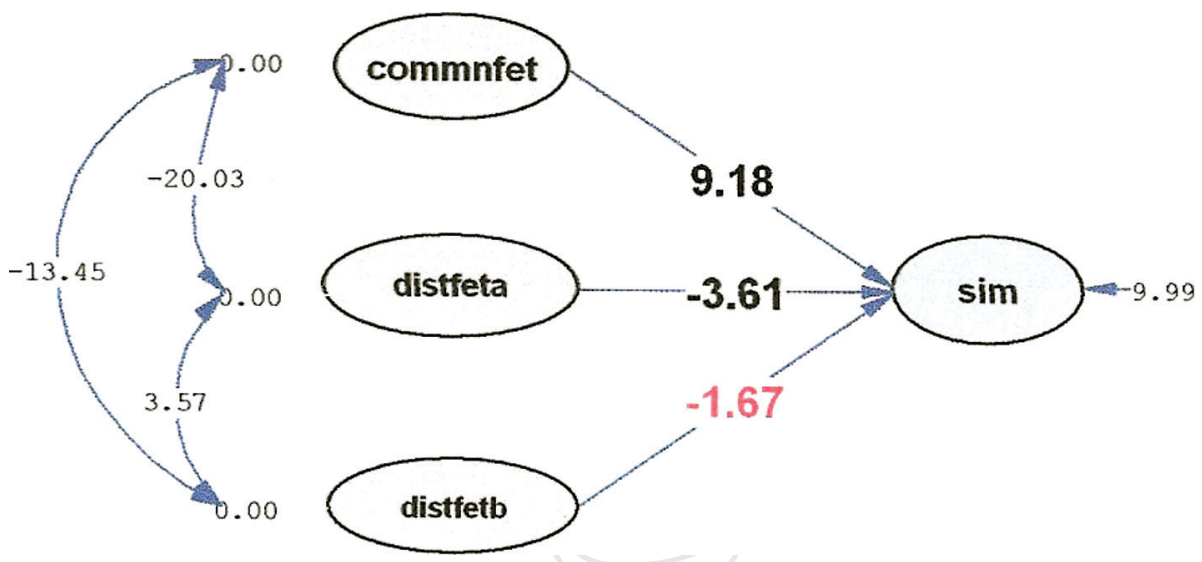

FIG. 2. Structural model of the modified contrast model with $t$ values or the path coefficients.

corresponding independent latent constructs in all the four measurement models were significant $(p<.01)$.

Extent of fit of the contrast model to sample data. The second research question is "To what extent does the contrast model fit human similarity judgments and features/attributes data for a sample of images?" The two-step approach of model testing (Jöreskog \& Sörbom, 1993; Schumacker \& Lomax, 1996) was followed. In the two-step approach, the measurement model is tested first to see if it holds for the set of observed and latent variables and to see if the observed $x$ variables measure their respective latent variables. The structural model is then tested once the measurement model holds.

The measurement models for the independent latent variables (common and distinctive features) already have been tested (see results of the first research question). Measurement models of independent latent variables with observed $x$ variables measured using Methods 1 and 3 and Methods 1 and 2 fit the data well. The measurement model, for the proposed model (Figure 1), with observed $x$ variables measured using Methods 1 and 3 is a poor fit, $\chi^{2}=22.18$, $d f=10, p<.05, R M S E A=.053$, SRMR $=.0185, G F I=$ $.987, A G F I=.955)$ compared to the measurement model with observed $x$ variables measured using Methods 1 and 2, $\chi^{2}=16.97, d f=10, p>.05, R M S E A=.040, S R M R=$ $.0205, G F I=.990, A G F I=.965)$. The latter has all five model fit indices that exceeded their recommended minimum levels $(G F I>.90$ and $A G F I>.90)$ or that are less than their recommended maximum levels (nonsignificant $\chi^{2}$ with $p>.05, R M S E A<.05, S R M R<.05)$. Therefore, the measurement model with observed $x$ variables measured using Methods 1 and 2 was used to test the contrast model.

A modified version of the contrast model, with observed $x$ variables measured using Methods 1 and 2 and the two observed $y$ variables (SIMAB and SIMBA), was tested. Not only did the model fit the sample data well, $\chi^{2}=16.97, d f=$ $10, p>.05, R M S E A=.040, S R M R=.0205, G F I=.990$, $A G F I=.965$ ), but all the loadings (parameter estimates) for the observed $x$ and $y$ variables onto their respective latent variables also were statistically significant $(p<.05)$. The path coefficients (except for the path coefficient for the structural relationship between distinctive features of $b$, distfetb, to perceived similarity, sim, with $p<.06$ ) in the structural model (Figure 2) with the three independent latent variables (commnfet, distfeta, and distfetb) and the latent dependent variable $(\operatorname{sim})$ were statistically significant $(p<.05)$. The major portion of the latent dependent variable's (sim) variance is explained $\left(R^{2}=.55\right)$ by the combination of the three independent latent variables (commnfet, distfeta, and distfetb). Furthermore, the direction of the path coefficients (positive path coefficients for common features and negative path coefficients for distinctive features) is proof that Tversky's (1977) statement that common features increase while distinctive features decrease the degree of similarity of pairs of objects holds. Therefore, it is fair to conclude that Tversky's contrast model provides a reasonably good fit to the data for a sample of images with path coefficients that are in the right direction and with the right magnitudes.

Relationships between perceived features and similarity of images. The third research question is "What is the relationship between perceived similarity of images, as judged by humans, and their features/attributes identified and described/listed by humans?" The main purpose of the study, by seeking an answer to this question, is to see whether more common features and less distinctive features result in higher similarity judgments. Pearson's product-moment correlation was used to determine the relationships between measures of perceived features (common and distinctive) and similarity judgments of images. As originally argued by Tversky (1977) and subsequently confirmed by Tversky and Gati (1978) and Johnson (1981, 1986), common features contribute to higher similarity judgments. Correlations between similarity judgments of the first set of pairs of images (SIMAB) and the three measures of their common features were found to be positive and statistically significant ( $r \mathrm{~s}=$ $.648, .513$, and $.675 ; p<.01)$. Likewise, the correlations 
between similarity judgments of the second set of pairs of images (SIMBA) and the three measures of their common features were positive and statistically significant as well $(r \mathrm{~s}=.624, .480$, and $.626 ; p<.01)$.

In contrast, distinctive features contribute to lower similarity ratings with correlations between similarity judgments of the first set of pairs of images (SIMBA) and measures of their distinctive features negative and statistically significant $(r s=-.507,-.296$, and $-.300 ; p<.01$, for the three measures of distinctive features of $a$, when $a$ and $b$ form a pair, respectively, and $r \mathrm{~s}=-.351,-.084$, and $-.097, p<.05$, for the three measures of distinctive features of $b$ ). Similar results hold for the correlations between similarity judgments of the second set of pairs of images (SIMBA) and the three measures of their distinctive features $(r s=-.460$, -.274 , and $-.277 ; p<.01$, for distinctive features of $a$, and $r \mathrm{~s}=-.375,-.091$, and $-.103, p<.05$, for distinctive features of $b$ ). Results of the structural equation analysis (model testing-Research Question 2) are in accord with those of the correlations where path coefficients between the independent latent variables (common and distinctive features) and the dependent latent variable (similarity) match correlation coefficients in both magnitude and direction. These results suggest that the more common features and the fewer distinctive features two images have, the more similar they are. Conversely, the fewer common features and the more distinctive features they have, the less similar they are. This is in total agreement with Tversky's (1977) assertion in formulating the contrast model, and these results are consistent with those from a similar research study in marketing by Johnson (1986), who used consumer products as materials.

Relative weights of common and distinctive features of images on their similarity. The fourth and final research question is "What are the relative weights given to common and distinctive features in human similarity judgments of images? To estimate the relative weights of common and distinctive features on similarity judgments, in addition to results from the testing of the contrast model, a regression analysis of similarity judgments on measures of common and distinctive features was conducted. In a way, the regression analysis results were used to validate results from structural equation modeling. The regression analyses results in Tables 3 and 4 confirm (except in the case of distinctive features measured using Method 3) the fact that common and distinctive features influence similarity of images in the expected direction and with the expected magnitudes (weights) as stated in Tversky's (1977) contrast model. Moreover, common and distinctive features reliably predict similarity of images.

Common features had the largest prediction power (weight), followed by distinctive features of $a$ (where $a$ and $b$ form a pair). Distinctive features of $b$ had the least weight. In other words, in the contrast model $[S(a, b)=\theta f(A \cap B)-$ $\alpha f(A-B)-\beta f(B-A)]$, for the sample of images in this study, $\theta>\alpha>\beta$. This is the case despite the fact that the
TABLE 3. Regression analysis of similarity judgments of the first set (SIMAB) on measures of common and distinctive features of images.

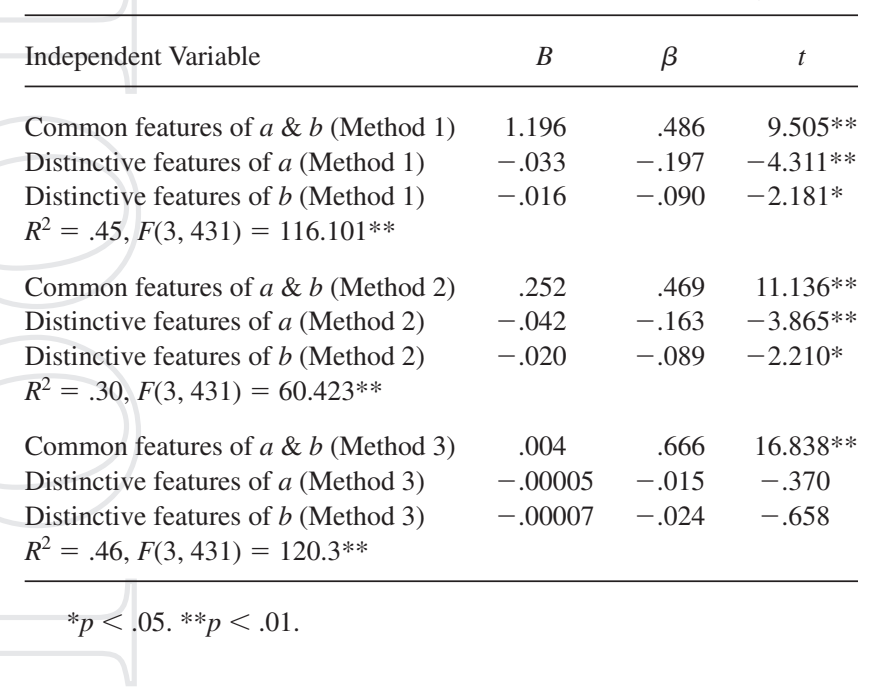

TABLE 4. Regression analysis of similarity judgments of the second set (SIMBA) on measures of common and distinctive features of images.

\begin{tabular}{|c|c|c|c|}
\hline Independent Variable & $B$ & $\beta$ & $t$ \\
\hline Common features of $a \& b$ (Method 1$)$ & 1.042 & .470 & $8.912 * *$ \\
\hline Distinctive features of $a($ Method 1) & -.023 & -.154 & $-3.268 * *$ \\
\hline Distinctive features of $b$ (Method 1) & -.020 & -.129 & $-3.036 * *$ \\
\hline \multicolumn{4}{|l|}{$R^{2}=.41, F(3,431)=100.913^{* *}$} \\
\hline Common features of $a \& b($ Method 2) & .213 & .440 & $10.199 * *$ \\
\hline Distinctive features of $a$ (Method 2) & -.035 & -.148 & $-3.438 * *$ \\
\hline Distinctive features of $b$ (Method 2) & -.019 & -.096 & $-2.310 *$ \\
\hline \multicolumn{4}{|l|}{$R^{2}=.26, F(3,431)=50.604^{* *}$} \\
\hline Common features of $a \& b($ Method 3$)$ & .003 & .617 & $14.79 * *$ \\
\hline Distinctive features of $a($ Method 3) & -.00004 & -.013 & -.303 \\
\hline Distinctive features of $b$ (Method 3) & -.00009 & -.035 & -.917 \\
\hline
\end{tabular}

$R^{2}=40, F(3,431)=93.285^{*} *$

$$
* p<.05 . * * p<.01 \text {. }
$$

participants were not asked to judge "how similar is image $a$ to image $b . "$ Tversky's (1977) assertion that features of the subject (i.e., the first object in the pair) are weighed more than those of the referent (i.e., the second object in the pair) holds. In the case of independent variables (common and distinctive features) measured using Method 3, even though the explained portions of variability in similarity (dependent variable) for both sets (.46 and .40, respectively) were significant, weights of distinctive features were nonsignificant $(p>.05)$. This is consistent with the findings of the tests of the measurement models for the independent latent variables where models with observed $x$ variables measured using Method 3 did not fit the sample data well. Furthermore, results of the regression analysis are in agreement with those of the structural equation analysis. Both the directions and magnitudes of loadings of independent latent constructs (common and distinctive features) on the dependent latent construct (similarity) are comparable to corresponding standardized regression weights $(\beta \mathrm{s})$. 


\section{Summary, Conclusions, and Future Research}

To reiterate, the main purpose of this study was to investigate the nature of the relationships between common and distinctive features of images and their similarity, using Tversky's (1977) contrast model as a theoretical framework, and to test the contrast model within the context of image representation and retrieval. This was achieved through correlation and regression analysis, and structural equation modeling. Four research questions were formulated and explored to address the main purpose of the study. The first two research questions address issues related to measures of common and distinctive features of images and measurement as well as structural model fit to the sample data. The remaining two research questions address the nature of the relationships between common and distinctive features (independent variables) and their similarity (dependent variable), including the prediction power (weights) of the independent variables.

Data were collected from 150 participants who were randomly assigned to two tasks $(n=75$ participants per task), an image description and a similarity judgment task, using a random sample of 30 images (435 pairs). The image description task data were summarized through content analysis and three measures of common and distinctive features. After initial screening and appropriate corrective measures, a set of 11 observed ( 9 observed $x$ and 2 observed $y$ ) variables and 4 latent constructs/variables were subjected to analysis using LISREL 8.54 (Jöreskog \& Sörbom, 1993).

In an indirect test of the appropriateness of the three methods of measuring the common and distinctive features of images, two measurement models, one with observed $x$ variables measured using Methods 1 and 3 and the other with observed $x$ variables measured using Methods 1 and 2 (see the methods section), fit the sample data well. Both the measurement model, with a combination of observed $x$ variables measured using Methods 1 and 2 and the two observed $y$ variables (SIMAB and SIMBA), and the structural model for the contrast model were tested and found to be a good fit to the data with statistically significant structural coefficients that have the right directions and magnitudes, and results confirm Tversky's (1977) contrast model. The third research question was meant to investigate the relationships/associations between common and distinctive features and similarity of images. The fact that common features contribute to higher similarity judgments while distinctive features have the opposite effect was substantiated by the results where the Pearson's product-moment coefficients for the correlations between measures of common features and similarity of images are positive and statistically significant $(p<.01$, one-tailed). The correlations between measures of distinctive features and similarity of images are negative and significant ( $p<.05$, one-tailed). Both regression analysis and structural equation modeling results confirm Tversky's contrast model in that common and distinctive features can reliably predict similarity of images and common features have more predictive power (weights) than do distinctive features.
In conclusion, attributes/features of documents are the basis for representation and indexing of both image and text documents. Not only does similarity play a central role in human perception and learning but psychological models of similarity also have been adopted for information-retrieval purposes in the form of similarity measures used for determining interdocument similarity or similarity between representations of documents and users' queries. However, few researchers devote their time and energy to trying to understand the nature of perceived features and similarity of documents, including image documents. This is the first study to test a psychological model of similarity, other than geometric models, in the context of document representation and retrieval. Results point to the fact that a linear combination of common and distinctive features of images can reliably predict their similarity, hence the relevance of the contrast model in the study of perceived features and similarity of objects, including images.

Results of this study have implications for researchers trying to better understand the nature of the relationships between perceived features of objects and their similarity as well as designers of information representation and retrieval systems. These results will provide the foundations for future research that will attempt to test the model using not only images but also other types of objects as stimuli. The study has methodological implications as well, especially the scaling/measurement of common and distinctive features and of perceived similarity. The reliable scaling procedures that were developed and used in this study are a significant methodological contribution to the field. Even though most of the current vector-space-model-based information retrieval systems use similarity measures derived from geometric models of similarity, despite their weaknesses, a similarity measure based on the contrast model would be a viable alternative; however, any similarity measure based on the contrast model needs to take into account the fact that common features carry more weight than do distinctive features. Moreover, while results of this study confirm the contrast model and similarity measures based on the model are suggested as viable alternatives to Minkowski metrics, another recent study (Neumann \& Gegenfurtner, 2006) demonstrated that current content-based image retrieval systems have the ability to narrow the semantic gap.

Researchers who attempt to further test the contrast model need to develop and introduce more measures of common and distinctive features as well as more scales for the measurement of similarity of objects. Subsequent studies should attempt to test the contrast model using samples of materials (i.e., images and other types of information objects) and participants (i.e., users of images and other information objects) selected from well-defined populations (e.g., a standard test collection of images and a community of image users such as photojournalists). The next logical step seems to be the design, development, and testing/evaluation of document representation methods and similarity measures based on the contrast model in a functional information retrieval environment. Testing may involve comparisons of 
the contrast model-based representation methods and similarity measures to existing methods and measures such as the vector-space model and the angle (cosine) and distancebased similarity measures. Traditional retrieval effectiveness and efficiency measures such as recall and precision as well as alternative metrics could be used for testing/evaluation purposes. Furthermore, if feature set-based document (especially image document) representations are to be adopted by retrieval systems, there is a need for thorough investigations into the types and levels of attributes/features not only perceived but also used during searching by human users.

\section{Acknowledgments}

I thank Dr. Linda Schamber for her patience, invaluable feedback, and great editing that improved this article considerably and the two anonymous reviewers for their careful review and constructive comments. I also thank Drs. Samantha Hastings, Brian O'Connor, and Gerald Knezek for their constant encouragement and feedback. To all participants of this study, I owe a great deal of gratitude.

\section{References}

Attneave, F. (1950). Dimensions of similarity. American Journal of Psychology, 63, 516-556.

Ben-Shakhar, G., \& Gati, I. (1992). The effect of common versus distinctive stimulus features on electrodermal orientation to significant stimuli. Psychophysiology, 29, 306-314.

Bruce, H. (1994). A cognitive view of the situational dynamism of usercentered relevance estimation. Journal of the American Society for Information Science, 45(3), 142-148.

Bruce, H. (1998). User satisfaction with information seeking on the Internet. Journal of the American Society for Information Science, 49(6), $541-556$.

Chu, H. (2001). Research in image indexing and retrieval as reflected in the literature. Journal of the American Society for Information Science and Technology, 52(12), 1011-1018.

Cohen, J. (1960). A coefficient of agreement for nominal scales. Educational and Psychological Measurement, 20, 37-46.

Cronbach, L.J. (1951). Coefficient alpha and the internal structure of tests Psychometrika, 16(3), 297-334.

Datta, R., Li, J., \& Wang, J.Z. (2005). Content-based image retrieval: Approaches and trends of the new age. In H. Zhang, J. Smith, \& Q. Tian (Eds.), Proceedings of the 7th ACM SIGMM international workshop on multimedia information retrieval (pp. 253-262). New York: ACM Press.

Dopkins, S., \& Ngo, C.T. (2001). The contribution of category relationships to similarity ratings. Canadian Journal of Experimental Psychology, 55, 244-252.

du Toit, M., \& du Toit, S. (2001). Interactive LISREL: User's guide. Lincolnwood, IL: Scientific Software International.

Eisenberg, M.B. (1986). Magnitude estimation and the measurement of relevance. Unpublished doctoral dissertation, Syracuse University, Syracuse, NY.

Eisenberg, M.B. (1988). Measuring relevance judgments. Information Processing \& Management, 24(4), 373-389.

Engen, T. (1971). Psychophysics II. Scaling methods. In J.W. Kling \& L.A. Riggs (Eds.), Woodworth \& Schlosberg's experimental psychology (pp. 47-86). New York: Holt, Rinehart \& Winston.

Gati, I., \& Tversky, A. (1984). Weighting common and distinctive features in perceptual and conceptual judgments. Cognitive Psychology, 16(3), 341-370.

Gay, R.L., \& Diehl, P.L. (1992). Research methods for business and management. New York: Macmillan.
Goldstone, R.L. (1999). Similarity. In R.A. Wilson \& F.C. Keil (Eds.), The MIT encyclopedia of the cognitive sciences (pp. 757-759). Cambridge, MA: MIT Press.

Gupta, A., Santini, S., \& Jain, R. (1997). In search of information in visual media. Communications of the ACM, 40(12), 35-42.

Holsti, O.R. (1969). Content analysis for the social sciences and humanities. Reading, MA: Addison-Wesley.

Hu, L., \& Bentler, P.M. (1999). Cutoff criteria for fit indexes in covariance structure analysis: Conventional criteria versus new alternatives. Structural Equation Modeling, 6, 1-55.

Johnson, M.D. (1981). Context effects in product perception. In K.B. Monroe (Ed.), Advances in consumer research (pp. 112-115). Chicago: Association for Consumer Research.

Johnson, M.D. (1986). Consumer similarity judgments: A test of the contrast model. Psychology \& Marketing, 3(1), 47-60.

Johnson, M.D., \& Horne, D.A. (1988). The contrast model of similarity and comparative advertising. Psychology \& Marketing, 5(3), 211-232.

Jöreskog, K.G. (1993). Testing structural equation models. In K.A. Bollen \& J.S. Long (Eds.), Testing structural equation models (pp. 294-316). Newbury Park, CA: Sage.

Jöreskog, K.G., \& Sörbom, D. (1999). PRELIS 2.3. Chicago: Scientific Software International.

Jörgensen, C. (1995). Image attributes: An investigation. Unpublished doctoral dissertation, Syracuse University, Syracuse, NY.

Jörgensen, C. (2003). Image retrieval: Theory and research. Lanham, MD: Scarecrow Press.

Kelloway, E.K. (1998). Using LISREL for structural equation modeling: A researcher's guide. Thousand Oaks, CA: Sage.

Lew, M.S., Sebe, N., Djeraba, C., \& Jain, R. (2006). Content-based multimedia information retrieval: State of the art and challenges. ACM Transactions on Multimedia Computing, Communications, and Applications, 2, 1-19.

Melara, R.D. (1992). The concept of perceptual similarity: From psychophysics to cognitive psychology. In D. Algom (Ed.), Psychophysical approaches to cognition (pp. 303-388). Amsterdam: North-Holland.

Neuendorf, K.A. (2002). The content analysis guidebook. Thousand Oaks, CA: Sage.

Neumann, D., \& Gegenfurtner, K.R. (2006). Image retrieval and perceptual similarity. ACM Transactions on Applied Perception, 3, 31-47.

Nunnally, J.C., \& Bernstein, I.H. (1994). Psychometric theory. New York: McGraw-Hill.

O'Connor, B.C., \& Wyatt, R.B. (2004). Photo provocations: Thinking in, with, and about photographs. Lanham, MD: Scarecrow Press.

Qin, J. (2000). Semantic similarities between a keyword database and a controlled vocabulary database: An investigation in the antibiotic resistance literature. Journal of the American Society for Information Science, 51(3), 166-180

Rosch, E. (1975). Cognitive representations of semantic categories. Journal of Experimental Psychology: General, 104(3), 192-233.

Rosch, E., \& Lloyd, B.B. (1978). Cognition and categorization. Hillsdale, NJ: Erlbaum.

Ross, R.T. (1934). Optimum orders for the presentation of pairs in the method of paired comparisons. Journal of Educational Psychology, 25, 375-383.

Rubner, Y. (1999). Perceptual metrics for image database navigation. Unpublished doctoral dissertation, Stanford University, Stanford, CA. Santini, S., \& Jain, R. (1999). Similarity measures. IEEE Transactions on Pattern Analysis and Machine Intelligence, 21(9), 871-883.

Schonlau, M., Fricker, R.D., \& Elliott, M.N. (2001). Conducting research surveys via e-mail and the Web. Santa Monica, CA: Rand.

Schumacker, R.E., \& Lomax, R.G. (1996). A beginner's guide to structural equation modeling. Mahwah, NJ: Erlbaum.

Shepard, R.N. (1962a). The analysis of proximities: Multidimensional scaling with an unknown distance function: I. Psychometrika, 27(2), 125-140.

Shepard, R.N. (1962b). The analysis of proximities: Multidimensional scaling with an unknown distance function: II. Psychometrika, 27(3), 219-246. 
Sloman, S.A., \& Rips, L.J. (1998). Similarity as an explanatory construct. In S.A. Sloman \& L.J. Rips (Eds.), Similarity and symbols in human thinking (pp. 1-15). Cambridge, MA: MIT Press.

Stevens, S.S. (1956). The direct estimation of sensory magnitudes-Loudness. American Journal of Psychology, 69(1), 1-25.

Stevens, S.S. (1966). A metric for the social consensus. Science, 151(3710), $530-541$.

Stevens, S.S. (1975). Psychophysics: Introduction to its perceptual, neural and social prospects. New York: Wiley.

Stricker, M., \& Orengo, M. (1995). Similarity of color images. In W. Niblack \& R. Jain (Eds.), Proceedings of the SPIE Conference on Storage and Retrieval for Image and Video Databases III (Vol. 2420, pp. 381-392). San Diego, CA.

Tabachnick, B.G., \& Fidell, L.S. (1989). Using multivariate statistics (2nd ed.). New York: HarperCollins.

Thurstone, L.L. (1927). A law of comparative judgment. Psychological Review, 34, 273-286.

Tversky, A. (1977). Features of similarity. Psychological Review, 84(4), 327-352.

Tversky, A., \& Gati, I. (1978). Studies of similarity. In E. Rosch \& B.B. Lloyd (Eds.), Cognition and categorization (pp. 79-98). Hillsdale, NJ: Erlbaum.

Tversky, A., \& Gati, I. (1982). Similarity, separability, and the triangle inequality. Psychological Review, 89(2), 123-154.
Ulhaque, E., \& Bahn, K.D. (1992). A spreading activation model of consumers' asymmetric similarity judgment. Advances in Consumer Research, 19(1), 782-786.

Weber, R.P. (1990). Basic content analysis (2nd ed.). Newbury Park, CA: Sage.

Weisberg, H.F. (1992). Central tendency and variability. Newbury Park, CA: Sage.

Zachary, J., Iyengar, S.S., \& Barhen, J. (2001). Content based image retrieval and information theory: A general approach. Journal of the American Society for Information Science and Technology, 52, 840-852.

Zachary, J.M. (2000). An information theoretic approach to content based image retrieval. Unpublished doctoral dissertation, Louisiana State University and Agricultural \& Mechanical College, Baton Rouge.

Zhang, J., \& Korfhage, R.R. (1999a). A distance and angle similarity measure method. Journal of the American Society for Information Science, 50(9), 772-778.

Zhang, J., \& Korfhage, R.R. (1999b). DARE: Distance and angle retrieval environment: A tale of the two measures. Journal of the American Society for Information Science, 50(9), 779-787.

Zhang, J., \& Rasmussen, E.M. (2001). Developing a new similarity measure from two different perspectives. Information Processing \& Management, 37(2), 279-294.

Zhu, B., \& Chen, H. (2000). Validating a geographical image retrieval system. Journal of the American Society for Information Science, 51(7), 625-634.

\section{Appendix A. Images Used in the Study}
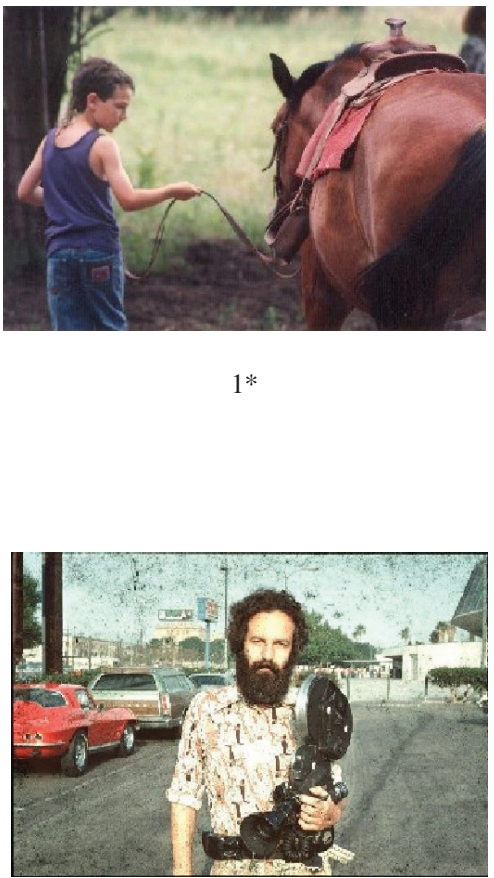

$4 *$

*O’Connor \& Wyatt (2004) (Used with permission).
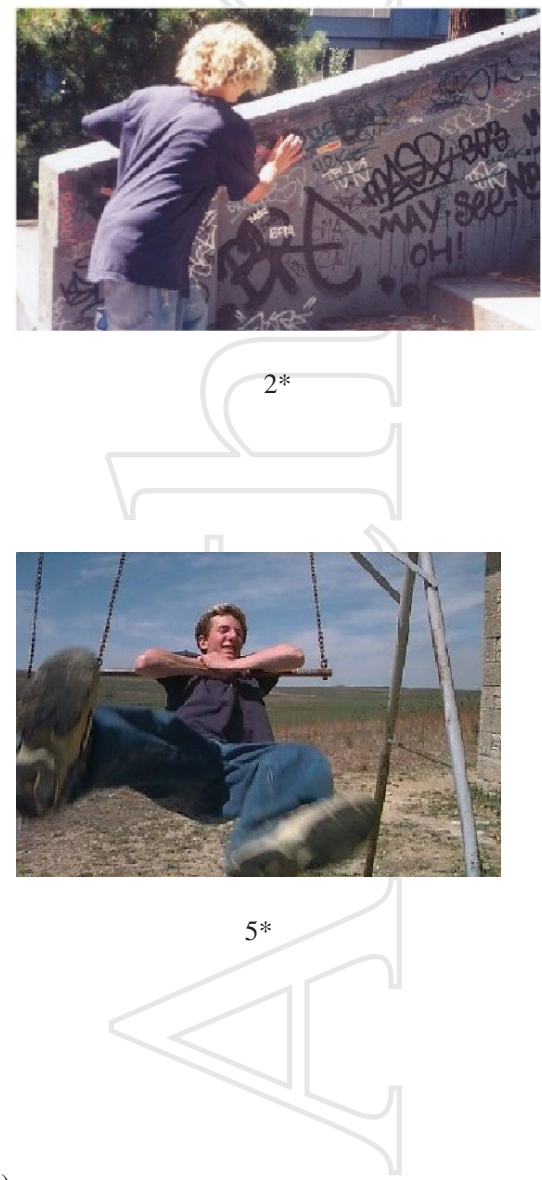

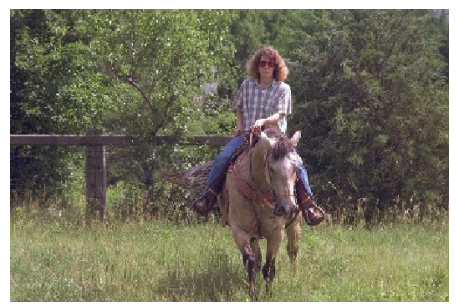

$3 *$

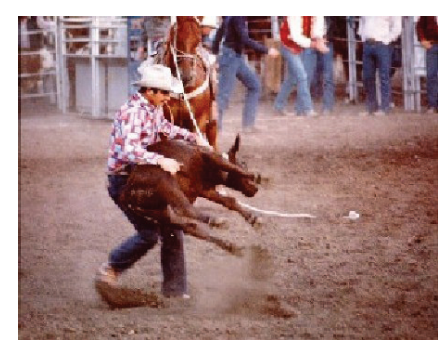

$6^{*}$ 
Appendix A. (Continued)

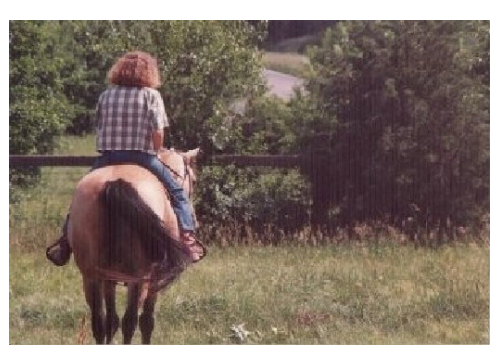

$7 *$

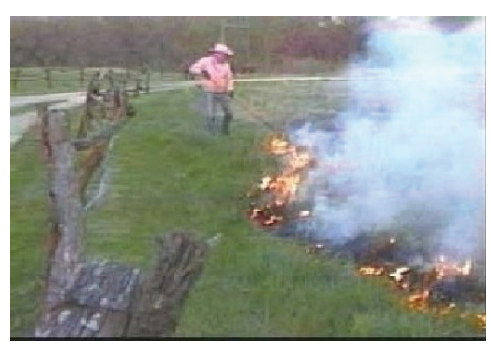

$10 *$

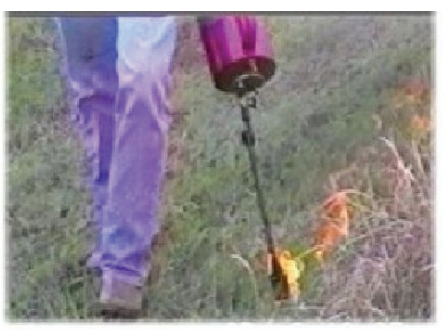

$13 *$

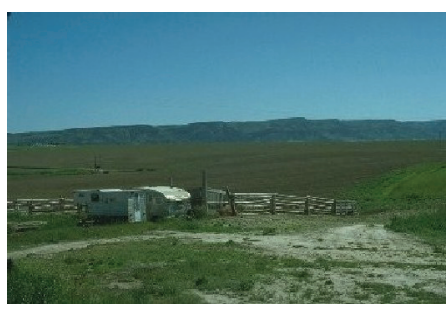

$16 *$

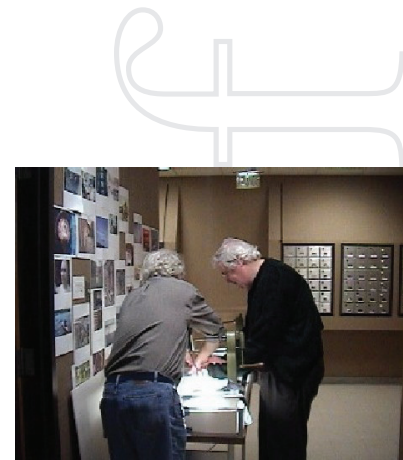

8*

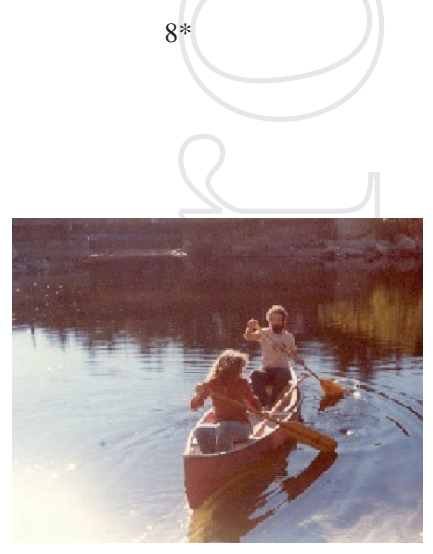

$11 *$

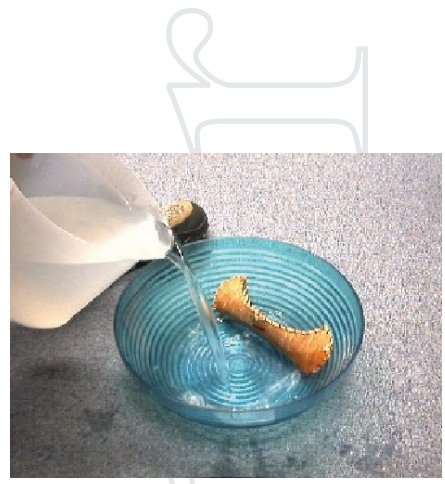

$14 *$
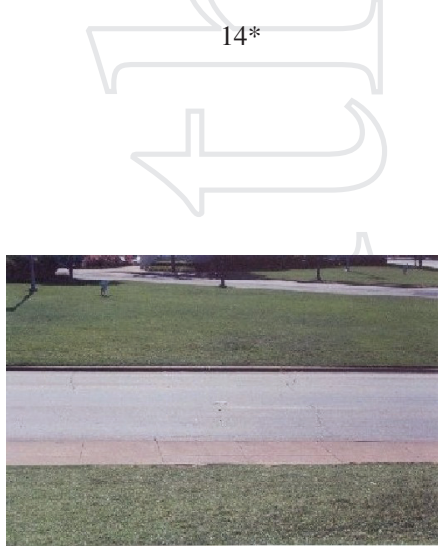

$17 *$

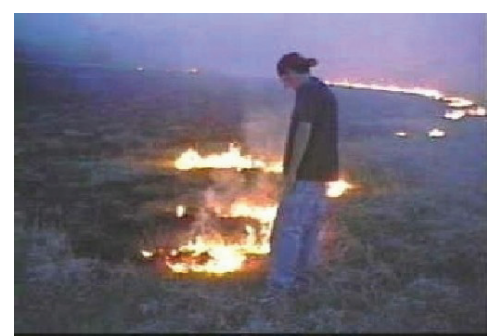

$9 *$

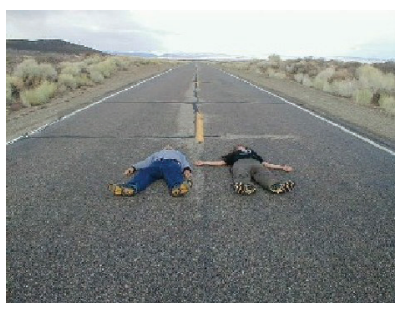

$12^{*}$

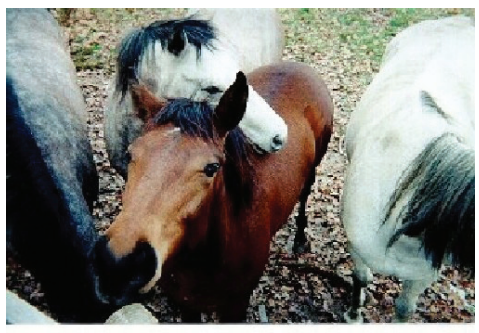

$15^{*}$

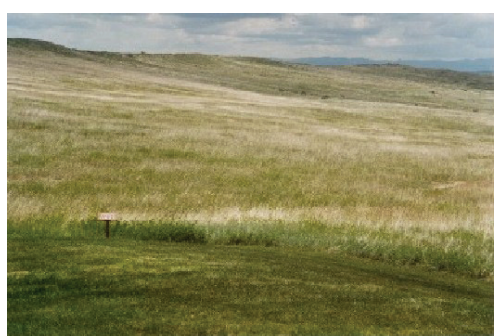

$18 *$

*O'Connor \& Wyatt (2004) (Used with permission). 
Appendix A. (Continued)

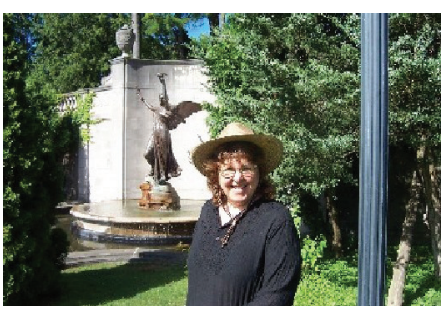

$19^{*}$

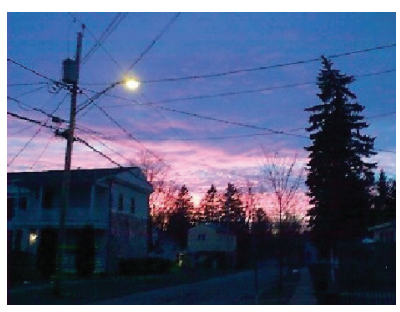

$22^{*}$

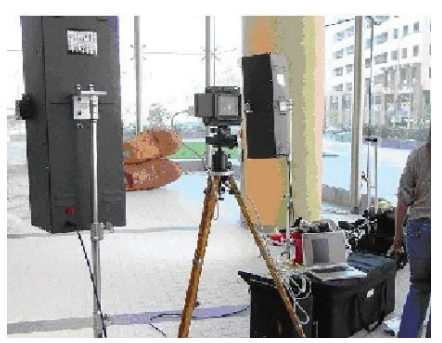

$25^{*}$

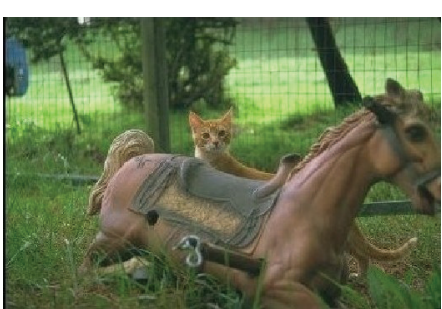

$28 *$

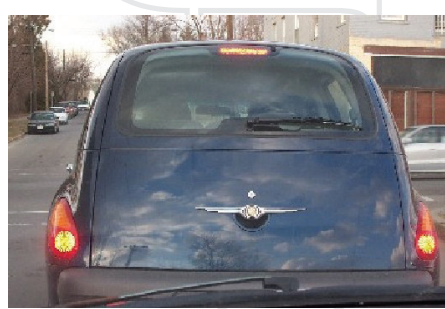

$20 *$

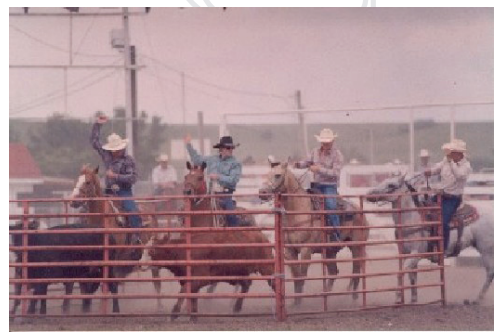

$23^{*}$

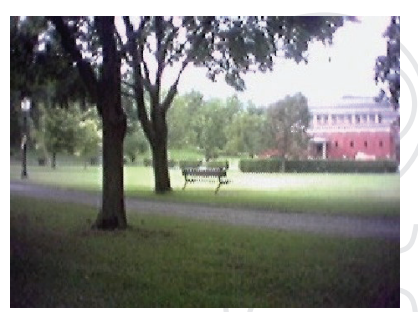

$26^{*}$

r
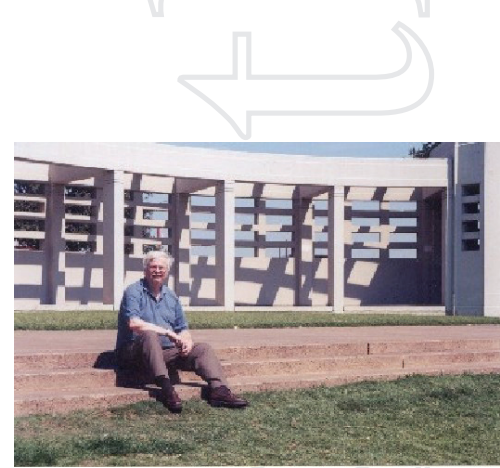

$29 *$

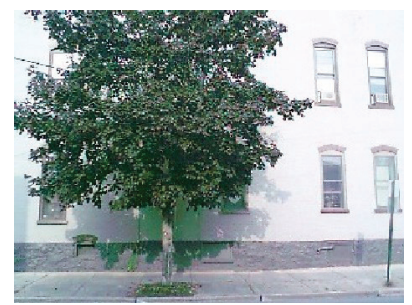

$21 *$

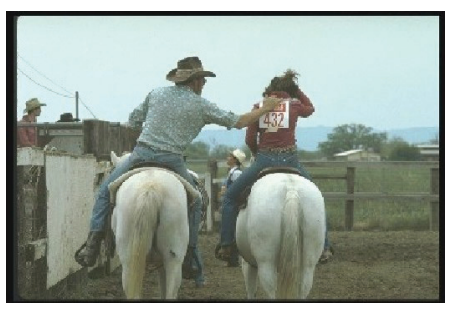

$24^{*}$

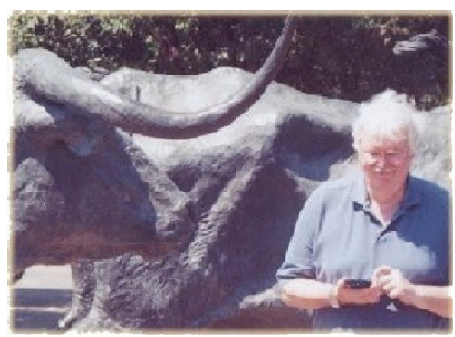

$27 *$

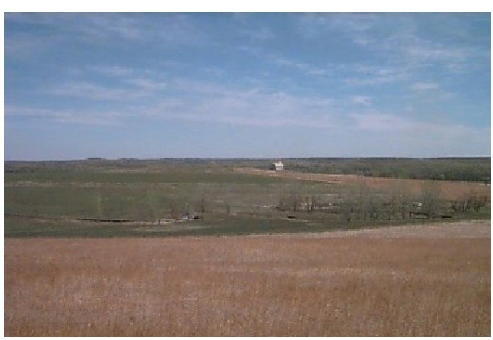

$30 *$

*O’Connor \& Wyatt (2004) (Used with permission). 


\section{Appendix B. Features Supplied by Participants for Image 24}

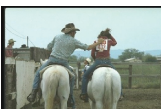

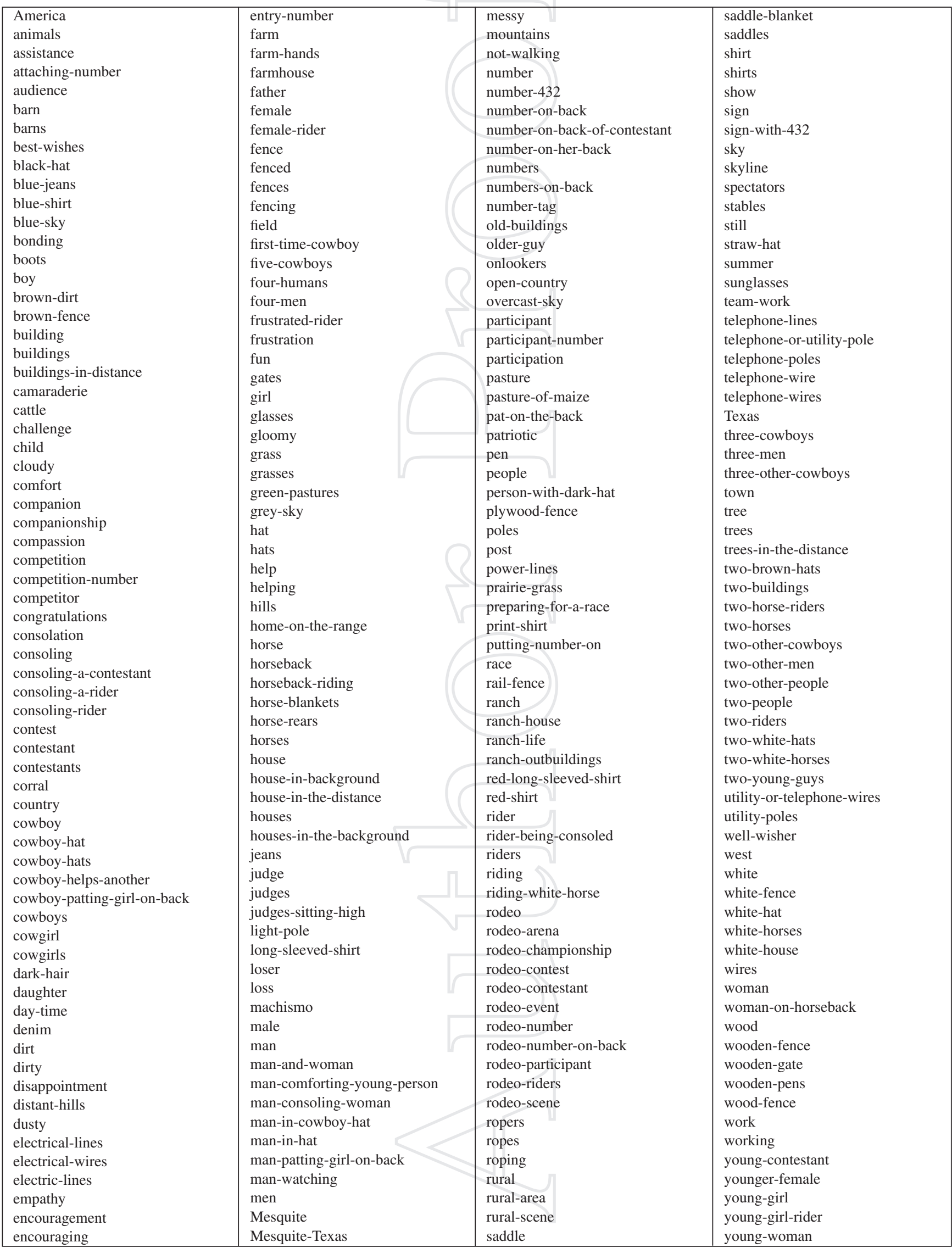




\section{AUTHOR QUERIES}

Note to author: Please check all equations carefully to make sure they have been typeset correctly.

1. Shepard (1964) is not in the References; please add a complete entry there.

2. Were [North Korea] and [Red China] in the original quote, or was they added in the present article for emphasis?

3. Does "he" refer to Tversky or to Rosch?
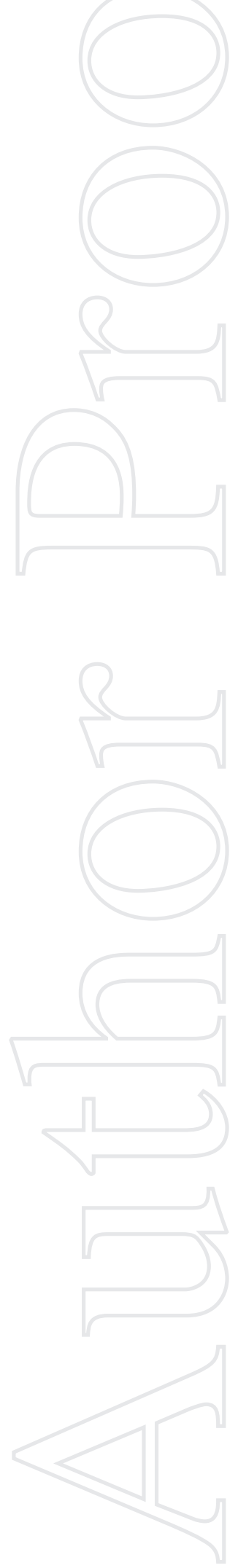